\title{
Monitoria de educação em saúde no ensino remoto: Um relato de experiência
}

\author{
Health education monitoring in remote education: An experience report \\ El seguimiento de la educación sanitaria en la educación a distancia: Un informe de experiencia
}

Recebido: 18/06/2021 | Revisado: 25/06/2021 | Aceito: 29/06/2021 | Publicado: 12/07/2021

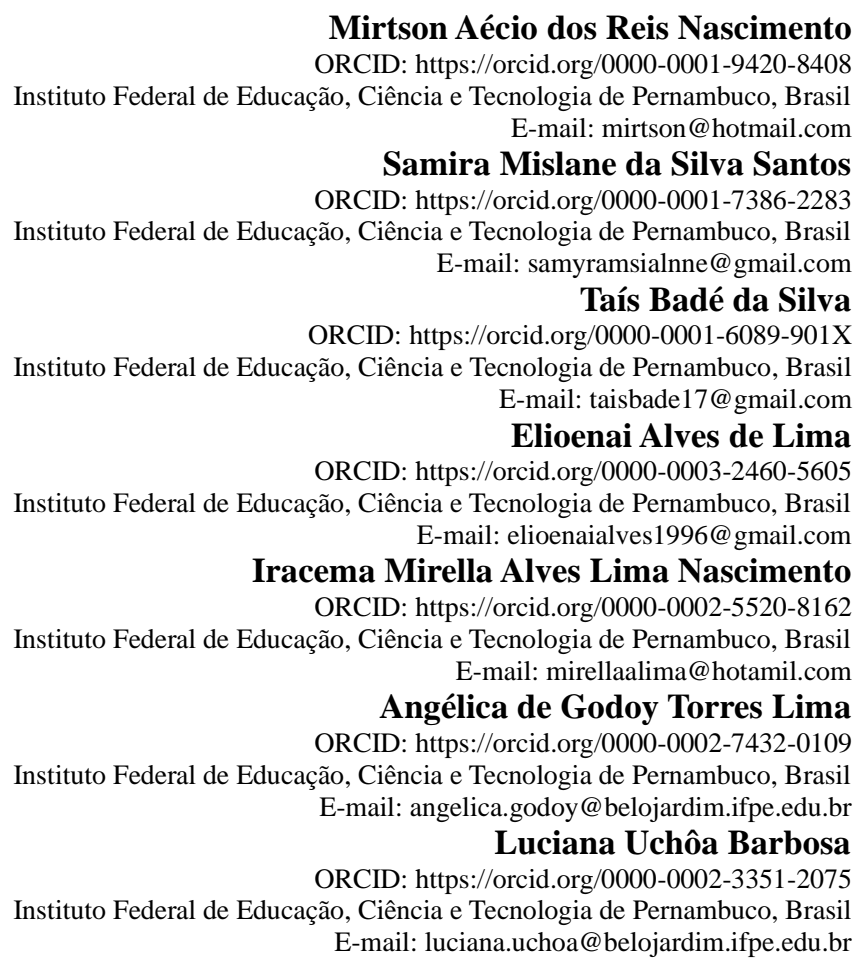

\section{Resumo}

O ensino remoto foi a forma de ensino adotada no Brasil para suprir a necessidade do distanciamento social, impulsionado pela pandemia COVID-19. Nesse contexto, o programa de monitoria ganha relevância ao ser entendido como uma ferramenta de apoio pedagógico. O objetivo deste trabalho é relatar as experiências da monitoria acadêmica, na modalidade remota, vivenciadas junto à disciplina de "Educação em Saúde", durante a pandemia COVID-19, em curso técnico em enfermagem, a partir da perspectiva do acadêmico monitor. Trata-se de um estudo descritivo, do tipo relato de experiência, fundamentado no relato das vivências de monitoria junto à disciplina de Educação em Saúde, no contexto do ensino remoto, de curso Técnico em Enfermagem em uma instituição federal de ensino no nordeste brasileiro. Foram utilizadas algumas ferramentas de comunicação para a execução das atividades, tais como o Google Classroom, Google Meet, Whatsapp e o Canva. As atividades realizadas pelos monitores abrangeram: levantamentos bibliográficos relacionados à "Educação em Saúde"; produção de materiais didáticos: Vídeo e Cartilha. Os monitores realizaram suas atividades de atendimento aos estudantes assistidos por meio de agendamento, reuniões no Google Meet e grupos de Whatsapp. A partir deste trabalho, verificou-se que, diante do panorama educacional, afetado pela pandemia COVID-19, a monitoria remota tornou-se um objeto essencial para o auxílio dos docentes no processo de ensino-aprendizagem, constituindo-se campo de conhecimento para todos os envolvidos no processo, professores, estudantes monitores e assistidos, além do estreitamento das relações interpessoais, mesmo em distanciamento físico.

Palavras-chave: Ensino; Educação em enfermagem; Tutoria; Educação em saúde; COVID-19.

\begin{abstract}
Remote learning was the form of teaching adopted in Brazil to meet the need for social distancing, driven by the COVID-19 pandemic. In this context, the monitoring program gains relevance when understood as a pedagogical support tool. The objective of this paper is to report the experiences of academic monitoring, in the remote modality, experienced in the discipline of "Health Education", during the COVID-19 pandemic, in a technical nursing course, from the perspective of the academic monitor. This is a descriptive study, of the experience report type, based on the report of the experiences of monitoring in the Health Education discipline, in the context of remote teaching, of a
\end{abstract}


Nursing Technician course in a federal educational institution in northeastern Brazil. Some communication tools were used to carry out the activities, such as Google Classroom, Google Meet, Whatsapp and Canva. The activities carried out by the monitors included: bibliographic surveys related to "Health Education"; production of teaching materials: Video and Primer. The monitors carried out their activities to assist students through scheduling, meetings on Google Meet and Whatsapp groups. From this work, it was found that, given the educational panorama, affected by the COVID-19 pandemic, remote monitoring has become an essential object to help teachers in the teaching-learning process, constituting a field of knowledge for all those involved in the process, teachers, monitors and assisted students, in addition to closer interpersonal relationships, even in physical distance.

Keywords: Teaching; Education nursing; Mentoring; Health education; COVID-19.

\section{Resumen}

El aprendizaje remoto fue la forma de enseñanza adoptada en Brasil para satisfacer la necesidad de distanciamiento social, impulsada por la pandemia de COVID-19. En este contexto, el programa de seguimiento gana relevancia cuando se lo entiende como una herramienta de apoyo pedagógico. El objetivo de este trabajo es reportar las experiencias de seguimiento académico, en la modalidad remota, vividas en la disciplina de "Educación en Salud", durante la pandemia de COVID-19, en un curso técnico de enfermería, desde la perspectiva del monitor académico. Se trata de un estudio descriptivo, del tipo relato de experiencia, basado en el relato de las experiencias de seguimiento en la disciplina de Educación para la Salud, en el contexto de la enseñanza a distancia, de un curso de Técnico en Enfermería en una institución educativa federal del noreste de Brasil. Se utilizaron algunas herramientas de comunicación para la realización de las actividades, como Google Classroom, Google Meet, Whatsapp y Canva. Las actividades realizadas por los monitores incluyeron: levantamientos bibliográficos relacionados con la "Educación para la Salud"; producción de material didáctico: Video y Primer. Los monitores llevaron a cabo sus actividades para ayudar a los estudiantes a través de la programación, reuniones en grupos de Google Meet y Whatsapp. A partir de este trabajo se encontró que, dado el panorama educativo, afectado por la pandemia del COVID-19, el monitoreo remoto se ha convertido en un objeto esencial para ayudar a los docentes en el proceso de enseñanza-aprendizaje, constituyéndose en un campo de conocimiento para todos los involucrados en el proceso de enseñanza-aprendizaje. proceso, profesores, monitores y alumnos asistidos, además de estrechar las relaciones interpersonales, incluso en la distancia física.

Palabras clave: Ensenãnza; Educación en enfermería; Tutoría; Educación en salud; COVID-19.

\section{Introdução}

A educação utiliza diversos recursos para auxiliar no processo de aprendizagem dos estudantes, para contribuir na construção de competências profissionais, visando o bom desempenho dos envolvidos. Neste contexto, pode-se destacar a monitoria acadêmica como um método que proporciona a construção de uma ponte entre estudante e conteúdo e através dessa estratégia o discente desenvolve interesse para aprofundar seus conhecimentos em um componente curricular. Sua finalidade não é apenas que esse aluno ensine, mas também, que se envolva no conteúdo trabalhado, de modo que seja fixado e associado o que aprendeu durante a vivência do componente curricular (Lima \& Pinheiro, 2018).

A monitoria é um programa de incentivo à formação acadêmica, que visa a ampliação dos espaços de aprendizagem, a melhoria da qualidade de ensino, o desenvolvimento da autonomia e a formação integral dos estudantes. Nesta perspectiva, o programa ganha relevância ao ser entendido como uma ferramenta de apoio pedagógico, pois o discente-monitor e o assistido têm a oportunidade de aprofundar conhecimentos em determinada matéria. Deste modo, fortalece habilidades teórico-práticas e esclarece dúvidas, sanando fragilidades inerentes a determinada área de conhecimento (Andrade et al., 2018).

As tarefas desempenhadas pelo estudante monitor consistem em dar apoio aos alunos da disciplina a qual está monitorando, apoiar na elaboração e na resolução de exercícios e projetos acadêmicos, ajudar na compreensão da bibliografia básica da disciplina, orientar quanto às dúvidas das matérias ministradas em sala e demais situações em que o professor orientador necessitar de auxílio (Gomes et al., 2020)

E por isso, para Gonçalves et al. (2021), a monitoria acadêmica é considerada uma ferramenta de auxílio no processo de ensino e aprendizagem, sua implementação permite contribuições tanto no âmbito do aprendizado e crescimento profissional quanto pessoal do discente e do docente. Consequentemente, constitui um espaço de troca experiências e descobertas, ao permitir o fortalecimento do relacionamento interpessoal entre professor e educando. 
O incentivo da realização de programas de monitoria no ensino técnico, promove um ganho intelectual para o monitor, contribuindo para o desenvolvimento dos monitorados e na troca de conhecimentos com o docente. Essa prática contribui para que o aluno obtenha oportunidade de realizar atividades que são comuns à docência e, consequentemente, desenvolve habilidades e torna-os mais responsáveis em suas atribuições (Lima \& Pinheiro, 2018).

$\mathrm{Na}$ perspectiva do ensino técnico em enfermagem, este programa oportuniza um maior desenvolvimento de habilidades técnicas e competências que favorece ao estudante um aprofundamento no campo da matéria monitorada. Os professores do componente curricular incentivam a participação dos alunos na monitoria, visto que o tempo durante as aulas é restrito e não permite a repetição dos procedimentos, abordados tantas vezes quanto necessário. Por esses fatores, a monitoria oferece subsídios para que o acadêmico possa desenvolver uma prática em enfermagem com maior segurança e precisão (Haag et al., 2008).

Presente no currículo do curso técnico em enfermagem, o componente curricular "Educação em Saúde" visa proporcionar a formação de técnicos em enfermagem comprometidos com os diferentes aspectos teóricos e filosóficos, que devem orientar o seu trabalho. A partir deste conhecimento, eles estarão aptos a desenvolver uma prática profissional convergente aos princípios da promoção da saúde, especialmente naqueles estabelecidos na Carta de Ottawa. Além disso, verifica a necessidade de atenção para os campos das habilidades pessoais e sociais, para o exercício da profissão que deve estar pautado no diálogo e numa educação popular libertadora (Salci et al., 2013).

Considerando o contexto da formação técnica em enfermagem, o ensino de educação em saúde exige a implementação de um processo teórico e prático, com vistas as exigências das políticas de educação e de saúde, bem como as demandas atuais do mercado de trabalho. Portanto, a inserção de atividades de Educação em Saúde na formação de estudantes da área da saúde contribui para uma prática social de promoção de saúde e consciência crítica-reflexiva das pessoas sobre sua situação de vida (Moreira, et al., 2019).

No âmbito do ensino do componente curricular "Educação em Saúde", a monitoria acadêmica no curso de Enfermagem é uma oportunidade necessária para que o monitor consolide conhecimentos teóricos e práticos, potencializados pela integração entre os próprios colegas, com os docentes e com a comunidade para a promoção da saúde e prevenção de doenças (Goulart et al., 2017). Diante do exposto, é possível perceber que as contribuições do monitor emergem com potencialidade e relevância na formação dos discentes que, com o docente, partilham experiências coletivas na construção do processo ensino-aprendizagem (Andrade et al., 2018).

Com o advento da pandemia em razão do COVID-19, as aulas presenciais foram substituídas por aulas em meios digitais, enquanto durar a situação de gerada pelo novo coronavírus, conforme disposto na Portaria $\mathrm{N}^{\circ} 343$, de 17 de março de 2020 (Diário Oficial da União, 2020). Nesse momento, a tecnologia por meio do ensino remoto vem favorecendo e possibilitando que as instituições de ensino possam vivenciar o processo de ensino e aprendizagem, embora esse momento venha sendo bastante desafiador para todos os envolvidos, com isso se tornou ainda mais necessário o apoio pedagógico através da monitoria (Gomes et al., 2020).

O ensino remoto emerge numa configuração do processo de ensino-aprendizagem denominada Educação Remota, isto é, práticas pedagógicas mediadas por plataformas digitais que se utiliza de aplicativos com os conteúdos, tarefas, notificações e/ou plataformas síncronas e assíncronas, como o Google Meet e Classroom (Alves, 2020).

Contudo, através da criação de uma Monitoria Virtual nesse espaço, o professor, o monitor estudante e os estudantes assistidos podem trocar ideias, experiências e construir uma solução para suas dúvidas de forma interativa e facilitada por uma ferramenta (Gomes et al., 2020).

Dessa forma, o objetivo deste trabalho foi relatar as experiências da monitoria acadêmica de estudantes de curso técnico em enfermagem, na modalidade remota, vivenciadas junto à disciplina de "Educação em Saúde", durante a pandemia 
COVID-19, a partir da perspectiva do acadêmico monitor, e descrever a contribuição da monitoria para a formação profissional em uma instituição federal de ensino.

\section{Metodologia}

$\mathrm{O}$ presente trabalho trata- se de um estudo descritivo, de natureza qualitativa, do tipo relato de experiência, desenvolvido por discentes e docentes do curso técnico em enfermagem de uma instituição federal de ensino do interior do Nordeste. A abordagem metodológica escolhida é justificada mediante a necessidade da interpretação, por parte dos pesquisadores, com suas opiniões sobre o fenômeno em estudo (Pereira et al., 2018). Deste modo, a pesquisa qualitativa não busca quantificar, mas por meio dela o pesquisador mantém contato direto e interativo com o objeto de estudo, direcionando o desenvolvimento de pesquisas que indiquem respostas para o problema elencado, em busca de entender, descrever e interpretar fatos (Proetti, 2017).

A experiência a ser descrita foi vivenciada por três estudantes monitores da disciplina de "Educação em Saúde", no contexto do ensino remoto, no período de 08 de outubro de 2020 a 20 de novembro de 2020. A disciplina integra o componente curricular do primeiro módulo do curso técnico em enfermagem, possui caráter obrigatório, com carga horária de 39 horas/aula e foi ministrada por dois docentes, na modalidade de ensino remoto. A mesma foi vivenciada através de momentos síncronos e assíncronos, utilizando as plataformas Google classroom, Google meet, Whatsapp que é um aplicativo de mensagens e o Canva, um editor versátil. Durante o período de aulas, encontravam-se matriculados 27 estudantes na disciplina.

Os métodos e recursos de ensino, utilizados nos momentos assíncronos do componente curricular, consistiram em orientações escritas, encaminhamentos de vídeos e artigos científicos para os estudantes assistidos. Já nos momentos síncronos, que tinham duração de 60 minutos, realizava-se a exposição teórica dialogada dos conteúdos, discussão de situações-problema utilizando histórias literárias (Jeca-tatu de Monteiro Lobato), discussão de artigos científicos e apresentação de seminários.

Durante as atividades, os estudantes matriculados na disciplina foram divididos em grupos, com a finalidade de realizar uma atividade com fins avaliativos, os mesmos deveriam produzir cartilhas educativas que abordassem temáticas voltadas para atenção primária à saúde, tais como arboviroses, parasitoses intestinais, higiene pessoal e contaminação de alimentos. As estratégias utilizadas para esta construção foram: consulta aos artigos científicos da área, aos cadernos de atenção básica do Ministério da Saúde e a ferramenta Canva.

Os estudantes monitores foram selecionados por meio de edital de seleção que utilizou o histórico escolar, escore acadêmico e análise de cartas de intenção para participação na monitoria. Por fim, foram selecionados dois voluntários e um estudante bolsista que dispunham de carga horária semanal para as atividades de monitoria variando entre 8 e 12 horas.

\section{Resultados e Discussão}

O ensino remoto trouxe consigo muitos desafios e exigiu dos estudantes e docentes a capacidade de adaptação a essa modalidade. Diante disso, no cenário de isolamento social, a atuação do estudante monitor nesta modalidade de ensino, promove a continuidade da prática de educacional, auxiliando no manejo das dificuldades vividas pelos estudantes assistidos ao cursar o componente curricular "Educação em saúde". Somado a isto, estão os novos processos exigidos pelo programa, para uma melhor adaptação ao ensino remoto, às práticas tecnológicas, à automotivação e à autonomia.

As atividades realizadas pelos monitores abrangeram o levantamento bibliográfico de temas relacionados ao componente curricular, produção de material didático, disponibilização de horários agendados para atendimento dos estudantes assistidos e orientação dos estudantes no planejamento das ações a serem implementadas. Todas as atividades dos monitores foram desenvolvidas sob supervisão direta dos docentes orientadores. 
Docentes de enfermagem tendem a buscar substituir práticas pedagógicas tradicionais por práticas mais dinâmicas, para tornar o aluno mais reflexivo e responsável por seu aprendizado. Sendo assim, a monitoria em curso técnico de enfermagem foi uma ação que contribuiu para a melhora no aprendizado dos monitorados, pois permite associação entre teoria e prática de maneira positiva, aproximação dos monitores com uma experiência semelhante à docência e maior contato dos alunos com publicações científicas, acrescendo no aprendizado de ambos (Belone et al., 2020).

As tarefas desempenhadas pelo discente monitor consistem em dar apoio aos estudantes da disciplina a qual está monitorando, apoiar na elaboração e na resolução de exercícios e projetos acadêmicos, ajudar na compreensão da bibliografia básica da disciplina, orientar quanto às dúvidas das matérias ministradas em aula e demais situações em que o professor orientador necessitar de auxílio (Souza, 2009). Cabe ressaltar que a elaboração de estudos e pesquisas, com o objetivo de esclarecer as dúvidas e os questionamentos dos estudantes monitorados, acaba por contribuir, de forma determinante, para a formação do seu espírito de pesquisador (Ventura, 2015).

Durante as aulas remotas, as ferramentas digitais tornaram-se os meios mais utilizados no processo de ensino, em que o Google Meet funcionou como uma sala de aula onde foi possível ter um maior contato com os discentes e o Whatsapp foi utilizado para esclarecer dúvidas e fornecer orientações sobre o trabalho exposto. A utilização do recurso digital Whatsapp permite a organização das atividades de maneira mais ágil e democrática, pois possibilita que informações importantes que antecedem a atividade sejam disponibilizadas em tempo real tais como autorizações, marcação de horários, confirmação de datas e compartilhamento de arquivos com fins pedagógicos dentro de um grupo no aplicativo (Purisco, 2020).

Diante da compreensão que o ensino sem autonomia é limitador, é necessário ao docente a utilização de diversos recursos no processo de ensino-aprendizagem, para que assim seja facilitado a compreensão do discente as informações que lhes são direcionadas, onde a tecnologia representa recursos que facilitam o acesso do aluno as informações e a possibilidade de maximização da aprendizagem de maneira autônoma (Oliveira \& Silva, 2015).

Para Oliveira e Silva (2015), a construção do ensino baseado na autonomia tem como prioridade a capacitação do estudante para investigar, selecionar, processar, assimilar, interpretar, procurando motivar a construção do saber, para que o discente possa através da autonomia buscar novos resultados, aprimorar sua capacidade, expor seus ideais, assim facilitando o processo de ensino-aprendizagem de qualidade, através da conscientização do aluno.

No decorrer das atividades, os monitores realizaram a orientação dos estudantes assistidos na elaboração de uma cartilha, através do aplicativo Canva. Para contribuir com a execução desta atividade, os estudantes monitores elaboraram um material didático com orientações acerca da construção de cartilhas, através de cinco passos, com informações sobre seu conceito, qual seu objetivo e como elaborá-la (Figura. 1). Outro produto gerado pelos monitores foi a produção de vídeos, apresentando o passo a passo de como utilizar as ferramentas digitais sugeridas. 
Figura 1: Material didático de orientação para elaboração de cartilhas.

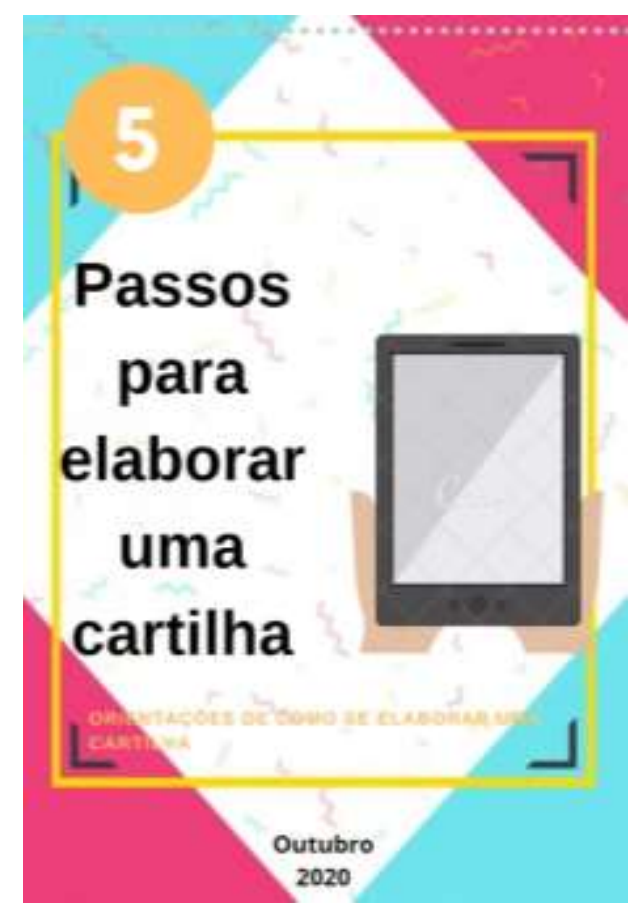

Fonte: Autores.

Os monitores perceberam um grau de dificuldade inicial em orientar os estudantes assistidos, a respeito da utilização do aplicativo Canva para construção da cartilha, pois o mesmo possui diversas ferramentas dificultando a orientação, o uso por meio dos alunos e principalmente pelo fato de os estudantes não estarem familiarizados com este tipo de ferramenta. Para solucionar os impasses identificados, foram utilizados diversos recursos como mensagens de texto, áudios, vídeos e até o próprio Canva, para ampliar o alcance da comunicação entre estudantes monitores e assistidos, visando uma maior eficácia nas ações.

Farias et al. (2018) relataram em seu trabalho que há diferentes níveis de maturidade quanto ao domínio do uso de tecnologias entre os participantes da pesquisa, especialmente no manuseio do smartphone enquanto artefato de uso para o estudo, o que prejudicou o progresso no processo de aprendizagem ativa por parte daqueles indivíduos com pouca experiência. Isso trouxe à luz que os desafios para utilização das tecnologias educacionais vão muito além do conhecimento acerca dos recursos e funcionalidades das ferramentas em si.

Precisa-se compreender que no começo é mais trabalhoso preparar um material para uma atividade a distância do que para uma atividade presencial. A introdução desta forma de aprendizado em cursos tradicionais ocorre em estágios, em que também é preciso conscientizar os educandos para que os mesmos também possam compreender a tecnologia como aliada aos estudos, requerendo um esforço para desenvolver certa autonomia para utilizar esses recursos (Gorgens \& Andrade, 2020).

$\mathrm{O}$ ensino remoto também trouxe consigo alguns impasses como a falta de formação específica para professores, o entendimento por parte da sociedade e o precário acesso da comunidade escolar a recursos tecnológicos, como computadores e internet de qualidade (Silva et al., 2020). Por outro lado, esse novo cenário proporcionou a participação mais ativa do estudante, baseado na dimensão relacional com o professor, buscando uma aprendizagem mais ativa. O estudante tornou-se o personagem principal e responsável no processo de aprendizagem (Oliveira et al., 2020).

Diante da realidade educacional fomentada pelo ensino remoto, Souza et al. (2021), sugerem que novos relatos de experiência sejam publicados, pois a partir dessas contribuições será possível conhecer diferentes estratégias de ensino que são desenvolvidas e implementadas nas instituições de educação. Consequentemente, este conhecimento poderá ser replicado em 
diferentes contextos educacionais do ensino na área da saúde.

\section{Considerações Finais}

A partir das atividades de monitoria realizadas observou-se o potencial dos monitores para a produção de material didático de maneira criativa e de fácil compreensão dos demais estudantes, o que demonstra os ganhos imensuráveis da monitoria, por meio da aprendizagem entre pares.

Destaca-se inicialmente, uma dificuldade com o uso da plataforma de design gráfico, Canva, portanto, é oportuno ressaltar a importância da oferta aos estudantes de cursos que possam aprimorar o uso das ferramentas digitais.

Pode-se inferir que a monitoria no formato remoto faz parte do novo cenário educacional em que estamos envolvidos, como mais uma ferramenta educacional dando suporte aos docentes no processo de ensino, além de permitir o protagonismo estudantil na construção do conhecimento. Apesar dos desafios, os monitores conseguiram vencer as dificuldades tornando-se mais autônomos e preparados para a atuação profissional.

Percebe-se que a monitoria de forma remota junto ao componente de Educação em saúde proporcionou uma vivência inédita para os estudantes, a construção de novas competências digitais aos discentes monitores e assistidos, bem como a habilidade de usá-las na promoção de educação para a saúde com mais autonomia. Sendo assim, a experiência da monitoria apresentou-se como uma oportunidade para desenvolvimento das habilidades profissionais e pessoais, pois possibilitou ampliar e consolidar conhecimentos, auxiliar na capacidade de interação entre os pares, proporcionando um aprendizado mútuo entre os discentes monitorados e o monitor.

Por fim, este relato demonstrou que a monitoria na modalidade remota foi uma experiência de grande aprendizado e descoberta para os professores e os estudantes envolvidos e que existem novas maneiras de ensinar e aprender. As limitações pertinentes a este estudo dizem respeito ao pouco tempo de coleta de dados. Em contrapartida, o presente trabalho sugere a necessidade de mais estudos voltados para o ensino técnico em enfermagem, especialmente no contexto remoto e pandêmico, no intuito de contribuir com a formação desta categoria profissional.

\section{Referências}

Alves, L. (2020). Educação remota: entre a ilusão e a realidade. Interfaces Científicas-Educação, 8(3), 348-365. https://doi.org/10.17564/23163828.2020v8n3p348-365

Andrade, E. G. R. D., Rodrigues, I. L. A., Nogueira, L. M. V., \& Souza, D. F. D. (2018). Contribuição da monitoria acadêmica para o processo ensinoaprendizagem na graduação em enfermagem. Revista Brasileira de Enfermagem, 71, 1596-1603. https://doi.org/10.1590/0034-7167-2017-0736

Belone, J. C. S, Pereira, J. D. C. N., Lima, A. D. G. T., \& Araújo, P. M. D. O. A. (2020). A monitoria acadêmica como intervenção teórico prática para estudantes do ensino técnico em enfermagem. Brazilian Journal of Development, 6(11), 86993-87004. https://doi.org/10.34117/bjdv6n11-212

Diário Oficial da União. Portaria n ${ }^{\circ} 343$, de 17 de março de 2020. Dispõe sobre a substituição das aulas presenciais por aulas em meios digitais enquanto durar a situação de pandemia do Novo Coronavírus - COVID-19. https://www.in.gov.br/en/web/dou/-/portaria-n-343-de-17-de-marco-de-2020-248564376

Farias, F. L. D. O., Brito, E. D. S., Melo, E. M. D., Bandeira, L. M. D. S. A., Costa, F. J. F. D., Diniz, I. J. D., \& Nunes, I. D. (2018). Práticas Pedagógicas Colaborativas utilizando Ferramentas Digitais: Um Relato de experiência na formação de educadores. Congresso Brasileiro de Informática na Educação, 7 ; Workshop de Informática na Escola, 24, 2018, Brasília. Anais eletrônicos do XXIV Workshop de Informática na Escola. Brasília: Sociedade Brasileira de Computação - SBC, 2018. http://dx.doi.org/10.5753/cbie.wie.2018.489

Gomes, M. A., de Sant'Anna, E. P. A., \& Maciel, H. M. (2020). Contexto atual do ensino remoto em tempos de COVID-19: um estudo de caso com estudantes do ensino técnico. Brazilian Journal of Development, 6(10), 79175-79192. http://dx.doi.org/10.34117/bjdv6n10-375

Gonçalves, M. F., Gonçalves, A. M., Fialho, B. F., \& Gonçalves, I. M. F. (2021). A importância da monitoria acadêmica no ensino superior. Práticas Educativas, Memórias e Oralidades-Rev. Pemo, 3(1), e313757-e313757. https://doi.org/10.47149/pemo.v3i1.3757

Gorgens, P., \& Andrade, P. C. (2020). A Educação Universitária Apoiadada Pelas Tecnologias Digitais De Informação E Comunicação. Revista Eletrônica Científica Ensino Interdisciplinar, 6(17). http://dx.doi.org/10.21920/recei72020617267280

Goulart, B. F., de Mello Barbosa, L. B., Bracarense, C. F., Rezende, M. P., Vicente, N. G., \& de Assis Simões, A. L. (2017). A monitoria de educação em saúde na enfermagem: relato de experiência. Revista de Enfermagem UFPE on line, 11(7), 2979-2984. https://doi.org/10.5205/1981-8963-v11i7a23479p29792984-2017 
Haag, G. S., Kolling, V., Silva, E., Melo, S. C. B., \& Pinheiro, M. (2008). Contribuições da monitoria no processo ensino-aprendizagem em enfermagem. Revista Brasileira de Enfermagem, 61(2), 215-220. https://doi.org/10.1590/S0034-71672008000200011

Lima, T. S., \& Pinheiro, S. S. (2018). A importância da monitoria acadêmica no desenvolvimento profissional do monitor: relato de experiência https://www.doity.com.br/anais/conexaofametro2018/trabalho/70746

Moreira, M. N., Silva, M. P. C., Duarte, A. P. G. M., Resende, M. P., Amaral, J. B. D., \& Contim, D. (2019). Educação em saúde no ensino de graduação em Enfermagem. Rev. enferm. Atenção, saúde, 61-70. https://doi.org/10.18554/reas.v8i1.3362

Oliveira N. C., \& Silva, A. L. B. (2015). Docência no ensino superior: o uso de novas tecnologias na construção da autonomia do discente. Saberes, Rolim de Moura, 3(2), 03-13. https://facsaopaulo.edu.br/wp-content/uploads/sites/16/2018/05/ed3/1.pdf

Oiveira, V. C., Neves, O. L., Martins, R. N., Santos, I. (2020). De Repente 4.0: Mudanças De Paradigma Educacional Em Tempo De Pandemia. In: Palú, J.; Schütz, J. A. \& Mayer, L. (2020). Desafios na educação em tempos de pandemia. Livro. https://www.researchgate.net/profile/JanetePalu/publication/349312858_DESAFIOS_DA_EDUCACA O_EM_TEMPOS_DE_PA NDEMIA/links/602a57259 2851c4ed571ff33/DESAFIOS-DAEDUCACAO-EM-TEMPOS-DE-PANDEMIA.pdf7

Pereira, A. S., Shitsuka, D. M., Parreira, F. J., \& Shitsuka, R. (2018). Metodologia da pesquisa científica. https://repositorio.ufsm.br/handle/1/15824

Proetti, S. (2018). As pesquisas qualitativa e quantitativa como métodos de investigação científica: Um estudo comparativo e objetivo. Revista Lumen-ISSN: 2447-8717, 2(4). http://www.periodicos.unifai.edu.br/index.php/lumen/article/view/60/88\#

Purisco, A. M. (2020). Educação digital 3.0: Uso pedagógico das tecnologias digitais de informação e comunicação na elaboração de sequências didáticas Monografia de Especialização, Universidade Federal de Minas Gerais, Escola de Educação Básica e Profissional, Centro Pedagógico, Belo Horizonte, MG, Brasil. https://repositorio.ufmg.br/handle/1843/35927

Salci, M. A., Maceno, P., Rozza, S. G., da Silva, D. M. G. V., Boehs, A. E., \& Heidemann, I. T. S. B. (2013). Educação em saúde e suas perspectivas teóricas: algumas reflexões. Texto \& Contexto Enfermagem, 22(1), 224-230. https://doi.org/10.1590/S0104-07072013000100027

Silva, L. A., Petry, Z. J. R., Uggioni, N., 2020. Desafios Da Educação Em Tempos De Pandemia: Como Conectar Professores Desconectados, Relato Da Prática Do Estado De Santa Catarina. In: Palú, J.; Schütz, J. A. \& Mayer, L. (2020). Desafios na educação em tempos de pandemia. Livro. https://www.researchgate.net/profile/Janete-Palu/publication/349312858_DESAFIOS_DA_EDUCACAO_EM_TEMPO S_DE_PANDEMIA/1 inks/602a57 259285 1c4ed571ff33/DESAFIOS-DA-EDUCACAO-EM-TEMPOS-DE-PANDEMIA.pdf

Souza, P. R. A. (2009) A importância da monitoria na formação de futuros professores universitários. Revista Âmbito Jurídico, XII, 61. https://ambitojuridico.com.br/edicoes/revista-61/a-importancia-da-monitoria-na-formacao-de-futuros-professores-universitarios/

Souza, M. S., Sousa, M. R. N., da Silva, L. A., Araújo, D. L., Nery, S. B. M., Júnior, J. E., \& Barbosa, L. C. L. (2021). Monitoria de enfermagem da disciplina de semiologia e semiotécnica: um relato de experiência. Research, Society and Development, 10(3), e37310313462-e37310313462. https://doi.org/10.33448/rsd-v10i3.13462

Ventura, A. F. (2015). Monitoria Acadêmica X Docência No Ensino Superior. Caderno De Graduação - Ciências Biológicas E Da Saúde - UNIT PERNAMBUCO, 2(1), 35-44. Recuperado de https://periodicos.set.edu.br/facipesaude/article/view/2950 